\title{
The Use of Fourier Transform Infrared Spectroscopy for Quantification of Adulteration in Virgin Walnut Oil
}

\author{
Pengjuan Liang, ${ }^{1}$ Hao Wang, ${ }^{1}$ Chaoyin Chen, ${ }^{1}$ Feng Ge, ${ }^{1}$ Diqiu Liu, ${ }^{1}$ Shiqi Li, ${ }^{2}$ \\ Benyong Han, ${ }^{1}$ Xianfeng Xiong, ${ }^{1}$ and Shenglan Zhao ${ }^{2}$ \\ ${ }^{1}$ Faculty of Life Science and Technology, Kunming University of Science and Technology, Kunming 650500, China \\ ${ }^{2}$ Faculty of Chinese Traditional Medicine, Yunnan University of Chinese Traditional Medicine, Kunming 650500, China \\ Correspondence should be addressed to Chaoyin Chen; chenchaoyin@163.com and Shenglan Zhao; zhaoshenglan@163.com
}

Received 23 June 2012; Accepted 19 November 2012

Academic Editor: Eugen Culea

Copyright (C) 2013 Pengjuan Liang et al. This is an open access article distributed under the Creative Commons Attribution License, which permits unrestricted use, distribution, and reproduction in any medium, provided the original work is properly cited.

Currently, the authentication of virgin walnut oil (VWO) has become very important due to the possible adulteration of VWO with cheaper plant oils such as soybean oil (SO), puer tea seed oil (PO), and sunflower oil (SFO). Methods involving Fourier transform infrared (FT-IR) spectroscopy combined with chemometric techniques (partial least square) were developed for quantification of $\mathrm{SO}, \mathrm{PO}$, and SFO in VWO. IR spectra of oil samples were recorded at frequency regions of $4000-650 \mathrm{~cm}^{-1}$ on horizontal attenuated total reflectance (HATR) attachment of FT-IR. PLS model correlates the actual and FT-IR estimated values of oil adulterants (SO, PO, and SFO) with coefficients of determination $\left(R^{2}\right)$ of $0.9958,0.9925$, and 0.9952 , respectively. The obtained RMSEC values of SO, PO, and SFO in VWO are 1.35\%, 1.85\%, and $1.43 \%(\mathrm{v} / \mathrm{v})$, respectively. The method, therefore, has potential as a rapid method for quantification of product adulteration.

\section{Introduction}

In the recent years, walnut oil has received great attention because of its biological activities and sensory qualities and has become a very important agricultural product for many countries. The unsaturated fatty acid in walnut oil is $90 \%$. There is about $47.4 \%$ of linoleic acid and $15.8 \%$ of linolenic acid [1]. Epidemiological studies show that walnut oil not only reduces serum cholesterol but at the same time has nutritional cranial nerve cells which can adjust plant nerve function. Other experts discovered that walnut oil does not only act as officinal, but also can be used as the "old man and the infant nutrition oil" as well as aerial work and flight personnel's senior health care oil [2]. Due to its higher price in the market, WO can be a target of adulteration with the cheaper oils such as soybean oil in order to gain economical profit. Therefore, the development of rapid and nonexpensive analytical techniques capable of detecting such adulterations in walnut oil is currently highly demanded.

In fact, the adulteration is a serious problem in trade of fats and oils for a long time, and it is of primary importance for consumers, food processors, and industries, because there is a great difference in quality and price for different oil products. The adulteration is increasingly more difficult to detect when the oil adulterant has similar chemical composition to the authentic oil $[3,4]$. In addition, chemical methods traditionally employed for the control of authenticity of virgin edible oil as gas chromatography and high performance liquid chromatography are expensive, time-consuming, require skilled operators, and have high environmental impact $[5,6]$.

New and complementary analytical techniques devoid of such troubles could act as supporting tools for currently used methods, being very helpful to improve the detection of VWO adulteration. Today, the application of Fourier transform infrared (FT-IR) spectroscopy has increased in food studies and particularly has become a powerful analytical tool in the study of edible oils and fats [7], because of its ability to serve as a "fingerprint technique," meaning that there are no two samples with the same FT-IR spectra, either in the number of peaks or in the maximum peak intensities [8]. IR spectroscopy can be taken into account as an ideal instrumental method for the authenticity studies of edible fats and oils. 
TABLE 1: The composition percentage for calibration and validation sets used in the binary mixtures of SO, PO, and SFO with VWO.

\begin{tabular}{|c|c|c|c|c|c|c|c|c|c|c|c|c|}
\hline \multirow{3}{*}{ Samples } & \multicolumn{4}{|c|}{ SO in VWO } & \multicolumn{4}{|c|}{$\mathrm{PO}$ in $\mathrm{VWO}$} & \multicolumn{4}{|c|}{ SFO in VWO } \\
\hline & \multicolumn{2}{|c|}{ Calibration } & \multicolumn{2}{|c|}{ Validation } & \multicolumn{2}{|c|}{ Calibration } & \multicolumn{2}{|c|}{ Validation } & \multicolumn{2}{|c|}{ Calibration } & \multicolumn{2}{|c|}{ Validation } \\
\hline & SO & VWO & SO & VWO & $\mathrm{PO}$ & VWO & $\mathrm{PO}$ & VWO & SFO & VWO & SFO & VWO \\
\hline 1 & 5 & 95 & 0 & 100 & 5 & 95 & 0 & 100 & 5 & 95 & 0 & 100 \\
\hline 2 & 8 & 92 & 2 & 98 & 8 & 92 & 2 & 98 & 8 & 92 & 2 & 98 \\
\hline 3 & 10 & 90 & 4 & 96 & 10 & 90 & 4 & 96 & 10 & 90 & 4 & 96 \\
\hline 4 & 13 & 87 & 6 & 94 & 15 & 85 & 6 & 94 & 15 & 85 & 6 & 94 \\
\hline 5 & 15 & 85 & 8 & 92 & 17 & 83 & 8 & 92 & 17 & 83 & 8 & 92 \\
\hline 6 & 20 & 80 & 10 & 90 & 20 & 80 & 10 & 90 & 20 & 80 & 10 & 90 \\
\hline 7 & 22 & 78 & 12 & 88 & 22 & 78 & 12 & 88 & 22 & 78 & 12 & 88 \\
\hline 8 & 25 & 75 & 16 & 84 & 25 & 75 & 16 & 84 & 25 & 75 & 16 & 84 \\
\hline 9 & 30 & 70 & 20 & 80 & 30 & 70 & 20 & 80 & 30 & 70 & 20 & 80 \\
\hline 10 & 35 & 65 & 23 & 77 & 35 & 65 & 23 & 77 & 35 & 65 & 23 & 77 \\
\hline 11 & 36 & 64 & 28 & 72 & 36 & 64 & 28 & 72 & 36 & 64 & 28 & 72 \\
\hline 12 & 40 & 60 & 32 & 68 & 40 & 60 & 32 & 68 & 40 & 60 & 32 & 68 \\
\hline 13 & 42 & 58 & 38 & 62 & 45 & 55 & 38 & 62 & 45 & 55 & 38 & 62 \\
\hline 14 & 45 & 55 & 42 & 58 & 48 & 52 & 42 & 58 & 48 & 52 & 42 & 58 \\
\hline 15 & 50 & 50 & 48 & 52 & 50 & 50 & 48 & 52 & 50 & 50 & 48 & 52 \\
\hline 16 & 100 & 0 & 50 & 50 & 100 & 0 & 50 & 50 & 100 & 0 & 50 & 50 \\
\hline
\end{tabular}

The presence of various spectroscopic sampling techniques such as attenuated total reflectance (ATR) and chemometric data evaluation software allows fast and reliable technique for authentication study of cod-liver oil (CLO) $[9,10]$. Using ATR, there is no excessive sample preparation; consequently, the use of hazardous solvents and reagents can be avoided [11]. For this reason, FT-IR spectroscopy and other vibrational spectroscopic techniques can be taken into consideration as "green analytical technique" for the analysis of edible fats and oils [12].

In addition, the chemometrics techniques have played a very important role in the study of edible fats and oils, especially for the authentication study [13]. Among such methods, partial least square (PLS) is a factorial multivariate calibration method that decomposes spectral data into loadings and scores, building the corresponding calibration models from these new variables $[14,15]$. This method, which requires analytes' compliance of Beer's Law, has been repeatedly coupled with FTIR spectroscopy and extensively used to obtain different quality parameters of edible oils [1621].

The aim of the present work is to develop a new application of the FTIR-PLS association as a rapid, inexpensive, and nondestructive authenticity measuring tool, useful to determine the adulteration of VWO with other edible oils and also to identify and quantify the percentage of the ruining agent in the blend. This approach represents a facile and convenient means for monitoring walnut oil quality with the advantage of ease of operation, high sample turnover, and no sample pretreatment.

\section{Materials and Methods}

Virgin walnut oil (VWO) and selected vegetable oils, namely soybean oil (SO), puer tea seed oil (PO), and sunflower oil (SFO) were purchased from the local market.

2.1. Quantification. For quantitative analysis, SO, PO, and SFO were mixed as binary mixture with VWO (each comprises 16 samples for calibration and 16 samples for validation). The concentration of each oils used in both calibration and validation is presented in Table 1. Each sample was subjected for FT-IR analysis.

2.2. Instrumentation. FT-IR spectra of samples were obtained using SHIMADZU FT-IR-8400 with HATR crystal of $\mathrm{ZnSe} 45^{\circ}$ equipped with deuterated triglycine sulphate (DTGS) as detector, potassium bromide $(\mathrm{KBr})$ as beam splitter, and controlled with the Omnic software (Version 6.0 Thermo Nicolet). The measurements were directly carried out by putting oil samples on HATR surface at controlled room temperature $\left(20^{\circ} \mathrm{C}\right)$ in IR region of $4000-650 \mathrm{~cm}^{-1}$, by accumulating 40 scans with the resolution of $4 \mathrm{~cm}^{-1}$. These spectra were subtracted from reference spectrum of air, acquired by collecting a spectrum from the cleaned blank HATR crystal before the measurement of each oil sample replication. The sample spectra were collected in triplicate and displayed as the average spectra. At the end of every scan, the surface of HATR crystal was cleaned with hexane twice and dried with special soft tissue, cleaned with acetone, and finally dried with soft tissue following the collection of each spectrum. 


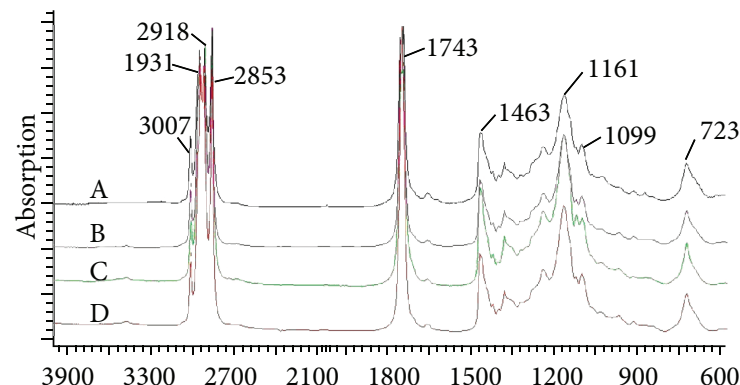

FIGURE 1: IR spectra of soybean oil (SO), puer tea seed oil (PO), sunflower oil (SFO), and virgin walnut oil (VWO) at IR region of $4000-650 \mathrm{~cm}^{-1}$. (A) VWO, (B) SO, (C) PO, and (D) SFO.

2.3. Chemometrics. The chemometric analyses were performed using the software TQ Analyst Version 6 (Thermo electron Corporation, Madison, WI). Quantification of adulterants (SO, PO, and SFO) in VWO was carried out using partial least square (PLS). Frequency regions for PLS were automatically selected by the software and were confirmed by investigating peaks where variations were observed. PLS calibration model was cross-validated using "leave-one-out" technique. This model was further used to predict the level of SO, PO, and SFO in independent samples in order to evaluate its predictive capability.

\section{Results and Discussion}

3.1. Spectra Analysis. Chemically, fats and oils are glycerol esterified with fatty acids. Some of the fats and oils might have quite similar composition; consequently, it is often difficult to detect adulteration of fats and oils physically [22]. The importance of IR spectroscopy in the identification of molecular structures originates from the much information content obtained and the possibility to assign certain absorption bands related to its functional groups. In fats and oils, most of the peaks and shoulders of the spectrum are attributable to the specific functional groups [17]. Because of its capability as a fingerprint technique, MIR spectroscopy allows one to differentiate authentic oils and those adulterated with others by observing the spectra changes due to the adulteration [23].

Figure 1 exhibits IR spectra of VWO, SO, PO, and SFO at frequency region of $4000-650 \mathrm{~cm}^{-1}$. The assignment of functional groups responsible for IR absorption is as follows: $3008 \mathrm{~cm}^{-1}$ (trans $=\mathrm{C}-\mathrm{H}$ stretch), $2954\left(-\mathrm{CH}_{3}\right.$ asymmetrical stretch), 2922 and 2853 (symmetrical and asymmetrical stretching of $\left.-\mathrm{CH}_{2}\right), 1743(-\mathrm{C}=\mathrm{O}$ stretch), 1654 (cis $-\mathrm{C}=\mathrm{C}$ stretch), 1463 ( $-\mathrm{CH}_{2}$ bending), 1417 ( $\mathrm{cis}=\mathrm{C}-\mathrm{H}$ bending), 1377 ( $-\mathrm{CH}_{3}$ bending), 1237 (-C-O stretch), 1160 (-C-O stretch; $-\mathrm{CH}_{2}$ bending), 1120 (-C-O stretch), 1098 (-C-O stretch), 1032 (-C-O stretch), 965 (trans $-\mathrm{CH}=\mathrm{CH}$ - bending out of plane), 871 (= $=\mathrm{CH}_{2}$ wagging), and $722 \mathrm{~cm}^{-1}$ (cis $-\mathrm{CH}=\mathrm{CH}$ - bending out of plane) $[24,25]$.

Taking into account the spectrum of VWO, SO, PO, and SFO, it can be seen that spectra of SO, PO, and SFO revealed some differences to $\mathrm{VWO}$, at frequency regions of $3007 \mathrm{~cm}^{-1}$ (attributed to the stretching vibration of cisvinylic), $2854 \mathrm{~cm}^{-1}$ due to asymmetrical and symmetrical stretching vibrations of $-\mathrm{CH}_{2}-, 1463 \mathrm{~cm}^{-1}$ because of the $-\mathrm{CH}_{2}$ bending, and $1098 \mathrm{~cm}^{-1}$ which are corresponding to $\mathrm{C}-\mathrm{O}$ stretching vibrations as well as at $722 \mathrm{~cm}^{-1}$ (cis $-\mathrm{CH}=\mathrm{CH}$ - bending out of plane). For this reason, these frequency regions in which the spectral variation was observed between VWO and vegetable oils (SO, PO, and SFO) were optimized to be exploited for quantification and classification of those in VWO.

3.2. Quantification of Vegetable Oils in VWO. The quantification of SO, PO, and SFO as adulterants in VWO was carried out using PLS algorithm. The spectral regions used for PLS calibration models are at 1463, 2854, and $3007 \mathrm{~cm}^{-1}$ for quantitative analysis of SO in VWO, at 723, 1099-1463, and $3007 \mathrm{~cm}^{-1}$ for quantification of PO in VWO, and at $723,1099-1464$, and $3007 \mathrm{~cm}^{-1}$ for quantification of SFO in VWO. The selection of these frequency regions was based on the optimization processes in which they offered the highest values of $R^{2}$ and the lowest values of error, either in calibration or in prediction models.

Figure 2 shows the PLS calibration model which correlates the actual and estimated purity quotient of VWO (\%v/v) obtained from FT-IR spectra at the specified regions. The difference between the actual and the observed concentration of VWO is relatively small with coefficients of determination $\left(R^{2}\right)$ values are $0.9958,0.9925$, and 0.9952 for adulterants (SO, PO and SFO), respectively. Root mean square error of calibration (RMSEC) was used to evaluate the error in calibration model. RMSEC value was calculated as follows:

$$
\text { RMSEC }=\sqrt{\frac{\sum_{i=1}^{n}(\text { actual }- \text { calculated })^{2}}{N-f-1}}[26] .
$$

The term "actual" refers to the known or true concentration of selected standards. Meanwhile the "calculated" or "predicted" refers to a value computed by the model using spectral data, where $N$ is the number of samples used in the calibration sets, and $f$ is number of factors used in the calibration model. The low value RMSEC indicates the good performance of PLS model. The obtained RMSEC values of VWO in SO, PO, and SFO are 1.35\%, 1.85\%, and $1.43 \%(\mathrm{v} / \mathrm{v})$, respectively.

In order to assess the prediction ability of the developed model, PLS calibration model was used to predict the levels of independent SO, PO, and SFO in VWO samples as validation or prediction datasets. The evaluation of the goodness of fit in the validation is performed by calculating the root mean square error of prediction (RMSEP) and $R^{2}$. RMSEP is calculated using the following equation:

$$
\text { RMSEP }=\sqrt{\frac{\sum_{i=1}^{m}(\text { actual }- \text { calculated })^{2}}{M-1}}[26] .
$$

Here $M$ is the number of samples used in the prediction sets. The values of $R^{2}$ are $0.9963,0.9961$, and 0.9978 for 


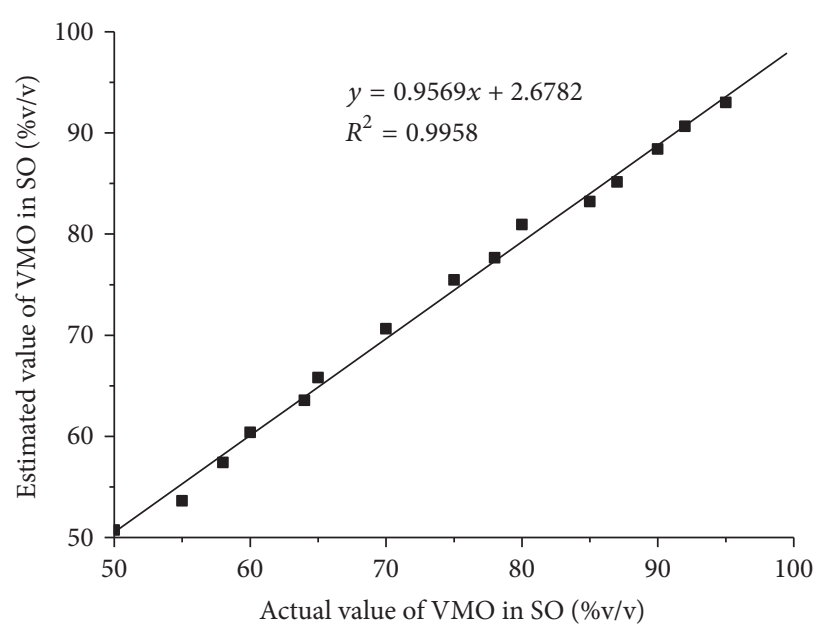

(a)

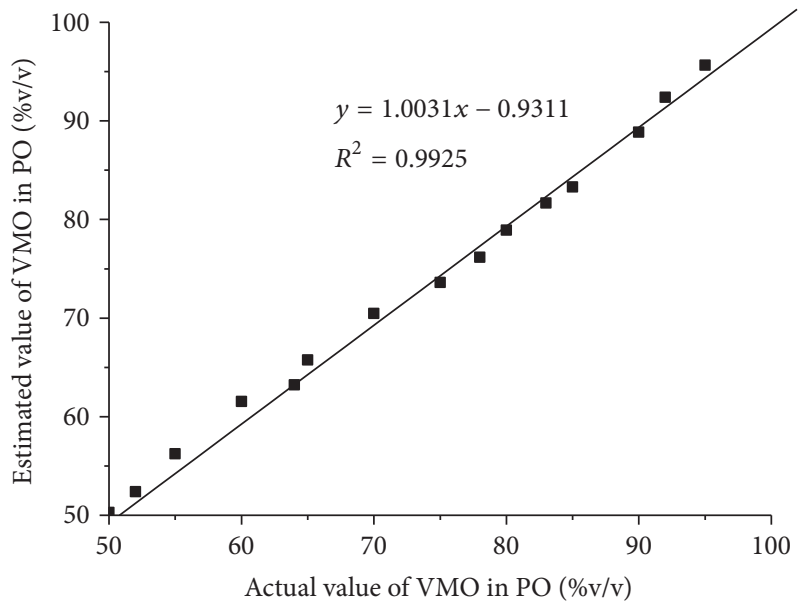

(b)

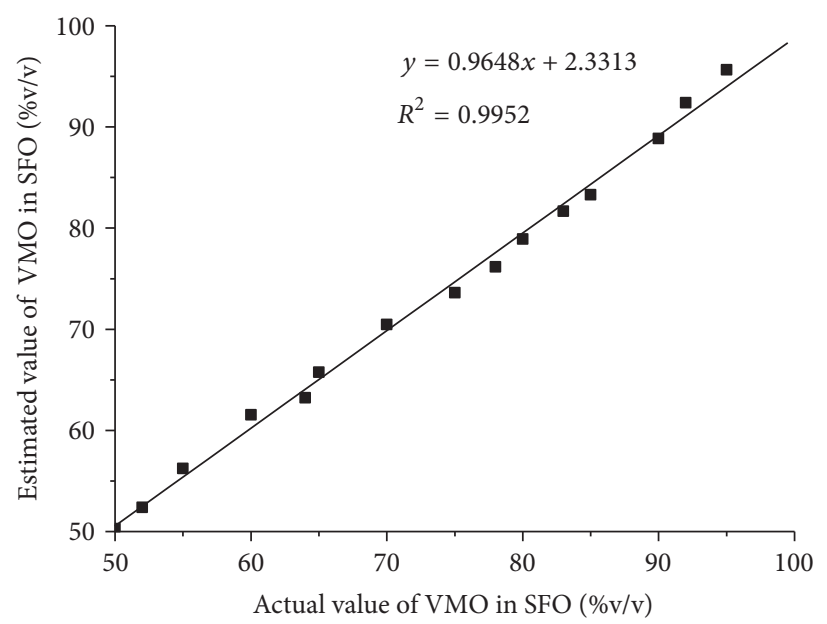

(c)

FIGURE 2: PLS calibration model for the relationship between actual and estimated concentrations of VWO in SO, PO, and SFO. (a) VWO in SO. (b) VWO in PO. (c) VWO in SFO.

TABLE 2: PLS performance for analysis of soybean oil, puer tea seed oil, and sunflower oil as oil adulterants in VWO.

\begin{tabular}{lccccc}
\hline Adulterants & Principal components & Calibration & $R^{2}$ & Prediction & RMSEC (\%v/v) \\
\hline Soybean oil & 5 & 0.9958 & 0.9963 & 1.35 & 1.31 \\
Puer tea seed oil & 5 & 0.9925 & 0.9961 & 1.85 & 1.31 \\
Sunflower oil & 5 & 0.9952 & 0.9978 & 1.43 & 1.11 \\
\hline
\end{tabular}

adulterants, Figure 3, respectively; meanwhile the RMSEP values are $1.31 \%, 1.31 \%$, and $1.11 \%(\mathrm{v} / \mathrm{v})$ for analysis of SO, $\mathrm{PO}$, and SFO as adulterants in VWO samples. The high values of $R^{2}$ and low values of RMSEP indicate the success of PLS regression model. Table 2 compiled PLS performance in terms of $R^{2}$, RMSEC, RMSEP, and the number of principal components for quantification of SO, PO, and SFO in VWO. The scatter plot for the relationship between actual and estimated concentration of SO, PO, and SFO in validation model is shown in Figure 3.
The developed PLS model was further evaluated by crossvalidation using "leave one out" technique. In this technique, one of the calibration samples is removed. Subsequently, the removed sample was predicted with the fashioned model using the residual samples and the procedure was repeated until each sample was excluded once from the model [27]. The values of root mean square error of cross-validation (RMSECV) obtained are relatively low, that is, $1.73 \%, 1.82 \%$, and $1.49 \%(\mathrm{v} / \mathrm{v})$, respectively, for SO, PO, and SFO. Based on this result, it can be stated that PLS appears to have a 


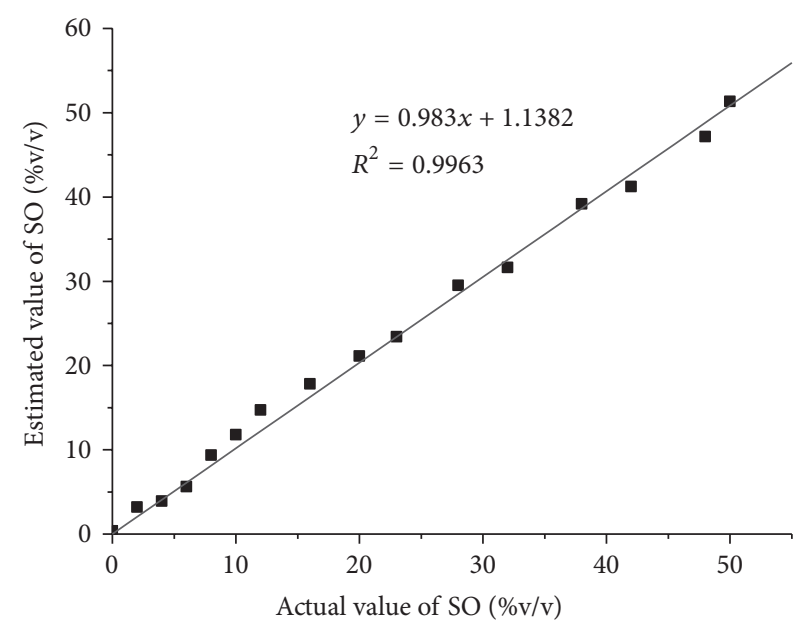

(a)

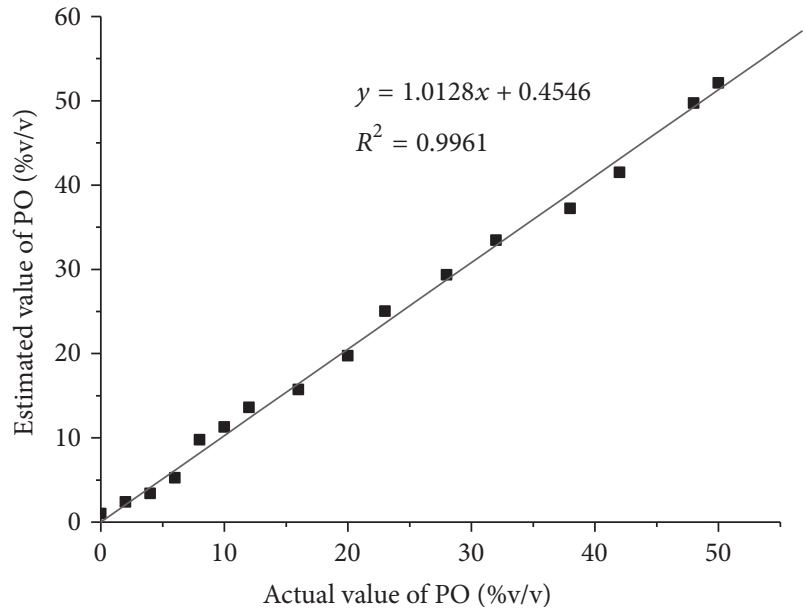

(b)

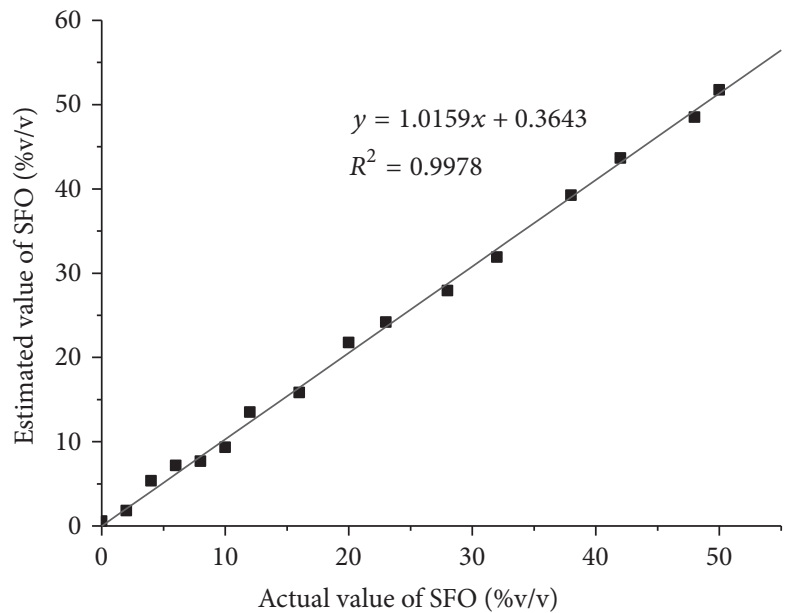

(c)

FIGURE 3: The relationship between actual and estimated concentrations of SO, PO, and SFO in VWO in validation model. (a) SO in VWO. (b) PO in VWO. (c) SFO in VWO.

reasonable ability to estimate the percentage of $\mathrm{SO}, \mathrm{PO}$, and SFO as oil adulterants in VWO samples.

\section{Conclusions}

It can be concluded that the adulteration of VWO with selected vegetable oils ( $\mathrm{SO}, \mathrm{PO}$, and $\mathrm{SFO}$ ) can be monitored with FT-IR spectroscopy using the ATR sample handling technique. PLS using normal spectra can be successfully used to detect the level of oil adulterants.

\section{Authors' Contribution}

P. Liang and H. Wang equally contributed to this work.

\section{Acknowledgments}

The authors gratefully acknowledge the financial support of State Science and Technology Support Program
(2011BAD46B00) and Yunnan Science and Technology Program (2011AB006).

\section{References}

[1] B. Y. Wan, H. Z. Dong, and H. Li, "Research on the properties and nutrition of walnut oil," China Western Cereals \& Oils Technology, vol. 26, pp. 18-19, 2001.

[2] S. L. Zhao, C. Y. Chen, and F. Ge, "Progressive study on functions and components," Journal of Yunnan University of Traditional Chinese Medicine, vol. 33, pp. 71-74, 2010.

[3] J. B. Rossell, B. King, and M. J. Downes, "Detection of adulteration," Journal of the American Oil Chemists' Society, vol. 60, pp. 333-339, 1983.

[4] A. K. Shukla, A. K. Dixit, and R. P. Singh, "Detection of adulteration in edible oils," Journal of Oleo Science, vol. 6, pp. 317-324, 2005.

[5] R. Aparicio and R. Aparicio-Ruíz, "Authentication of vegetable oils by chromatographic techniques," Journal of Chromatography A, vol. 881, no. 1-2, pp. 93-104, 2000. 
[6] W. Kamm, F. Dionisi, C. Hischenhuber, and K. H. Engel, "Authenticity assessment of fats and oils," Food Reviews International, vol. 17, no. 3, pp. 249-290, 2001.

[7] M. D. Guillen and N. Cabo, "Some of the most significant changes in the Fourier transform infrared spectra of edible oils under oxidative conditions," Journal of the Science of Food and Agriculture, vol. 80, pp. 2028-2036, 2000.

[8] D. L. Pavia, G. M. Lampman, and G. S. Kriz, Introduction to Spectroscopy, Thomson Learning, Victoria, Australia, 3rd edition, 2001.

[9] L. M. Reid, C. P. O’Donnell, and G. Downey, "Recent technological advances for the determination of food authenticity," Trends in Food Science \& Technology, vol. 17, pp. 344-353, 2006.

[10] A. A. Christy, P. K. Egeberg, and E. T. Østensen, "Simultaneous quantitative determination of isolated trans fatty acids and conjugated linoleic acids in oils and fats by chemometric analysis of the infrared profiles," Vibrational Spectroscopy, vol. 33, no. 1-2, pp. 37-48, 2003.

[11] L. E. Rodriguez-Saona and M. E. Allendorf, "Use of FTIR for rapid authentication and detection of adulteration of food," Annual Review of Food Science and Technology, vol. 2, no. 17, pp. 1-17, 2011.

[12] J. Moros, S. Garrigues, and M. de la Guardia, "Vibrational spectroscopy provides a green tool for multi-component analysis," Trends in Analytical Chemistry, vol. 29, no. 7, pp. 578-591, 2010.

[13] I. S. Arvanitoyannis and A. Vlachos, "Implementation of physicochemical and sensory analysis in conjunction with multivariate analysis towards assessing olive oil authentication/adulteration," Critical Reviews in Food Science and Nutrition, vol. 47, no. 5, pp. 441-498, 2007.

[14] P. Geladi and B. R. Kowalski, "Partial least-squares regression: a tutorial," Analytica Chimica Acta, vol. 185, pp. 1-17, 1986.

[15] H. Martens and T. Næs, Multivariate Calibration, Wiley, Chichester, UK, 1989.

[16] A. Al-Alawi, F. R. Van de Voort, and J. Sedman, "New FTIR method for the determination of FFA in oils," Journal of the American Oil Chemists' Society, vol. 81, no. 5, pp. 441-446, 2004.

[17] A. Bendini, L. Cerretani, F. Di Virgilio, P. Belloni, M. BonoliCarbognin, and G. Lercker, "Preliminary evaluation of the application of the ftir spectroscopy to control the geographic origin and quality of virgin olive oils," Journal of Food Quality, vol. 30, no. 4, pp. 424-437, 2007.

[18] E. Bertran, M. Blanco, J. Coello, H. Iturriaga, S. Maspoch, and I. Montoliu, "Determination of olive oil free fatty acid by Fourier transform infrared spectroscopy," Journal of the American Oil Chemists' Society, vol. 76, no. 5, pp. 611-616, 1999.

[19] F. A. Iñón, J. M. Garrigues, S. Garrigues, A. Molina-Díaz, and M. de la Guardia, "Selection of calibration set samples in determination of olive oil acidity by partial least squaresattenuated total reflectance-Fourier transform infrared spectroscopy," Analytica Chimica Acta, vol. 489, no. 1, pp. 59-75, 2003.

[20] H. Li, F. R. Van De Voort, A. A. Ismail, and R. Cox, "Determination of peroxide value by Fourier transform near-infrared spectroscopy," Journal of the American Oil Chemists' Society, vol. 77, no. 2, pp. 137-142, 2000.

[21] H. Li, F. R. Van De Voort, A. A. Ismail et al., "Discrimination of edible oil products and quantitative determination of their iodine value by Fourier transform near-infrared spectroscopy," Journal of the American Oil Chemists' Society, vol. 77, no. 1, pp. 29-36, 2000.
[22] A. A. Christy, S. Kasemsumran, Y. Du, and Y. Ozaki, "The detection and quantification of adulteration in olive oil by nearinfrared spectroscopy and chemometrics," Analytical Sciences, vol. 20, no. 6, pp. 935-940, 2004.

[23] K. Y. L. Yap, S. Y. Chan, and C. S. Lim, "Infrared-based protocol for the identification and categorisation of ginseng and its products," Food Research International, vol. 40, pp. 643-652, 2007.

[24] M. D. Guillén and N. Cabo, "Characterization of edible oils and lard by fourier transform infrared spectroscopy: relationships between composition and frequency of concrete bands in the fingerprint region," Journal of the American Oil Chemists' Society, vol. 74, no. 10, pp. 1281-1286, 1997.

[25] M. J. Lerma-García, G. Ramis-Ramos, J. M. Herrero-Martínez, and E. F. Simó-Alfonso, "Authentication of extra virgin olive oils by Fourier-transform infrared spectroscopy," Food Chemistry, vol. 118, no. 1, pp. 78-83, 2010.

[26] M. M. Paradkar and J. Irudayaraj, "A rapid FTIR spectroscopic method for estimation of caffeine in soft drinks and total methylxanthines in tea and coffee," Journal of Food Science, vol. 67, no. 7, pp. 2507-2511, 2002.

[27] G. Gurdeniz, F. Tokatli, and B. Ozen, "Differentiation of mixtures of monovarietal olive oils by mid-infrared spectroscopy and chemometrics," European Journal of Lipid Science and Technology, vol. 109, no. 12, pp. 1194-1202, 2007. 

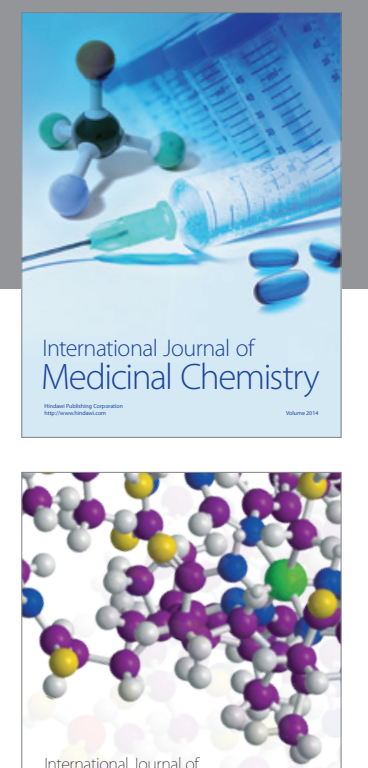

\section{Carbohydrate} Chemistry

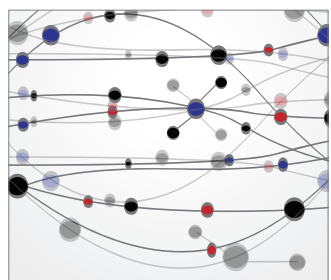

The Scientific World Journal
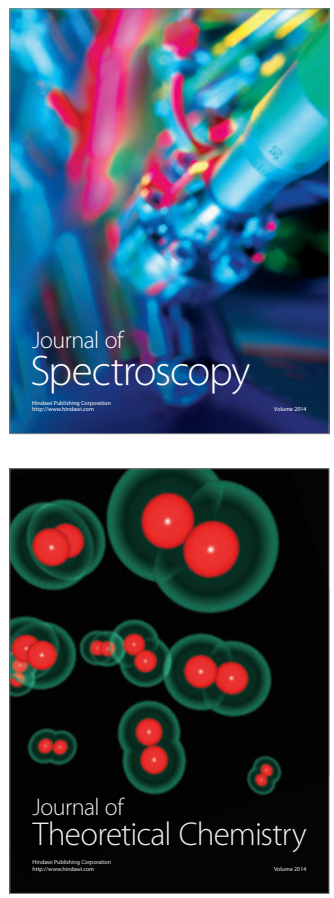
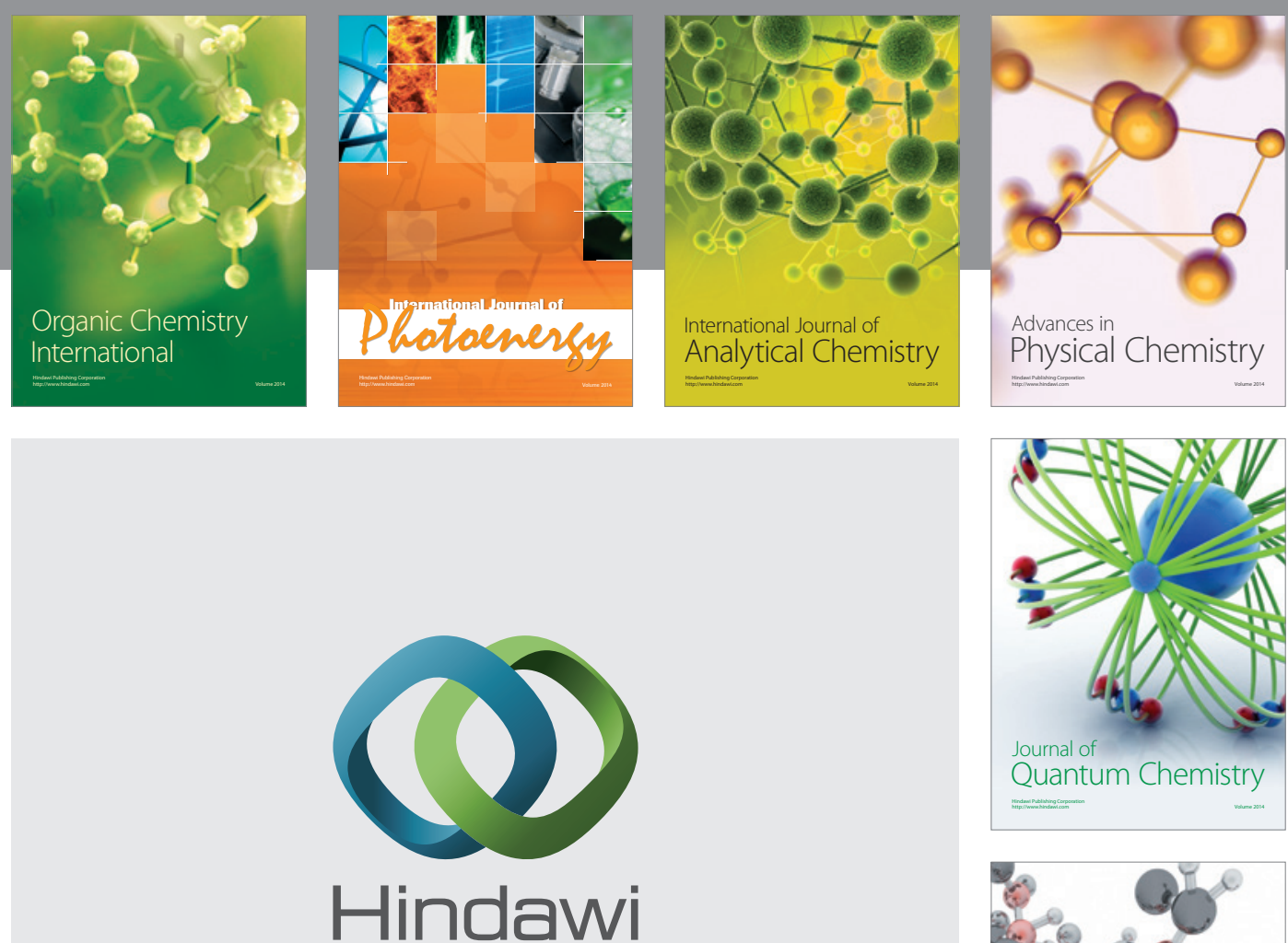

Submit your manuscripts at

http://www.hindawi.com

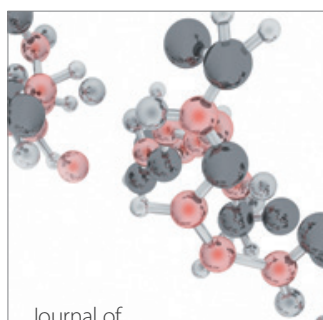

Analytical Methods

in Chemistry

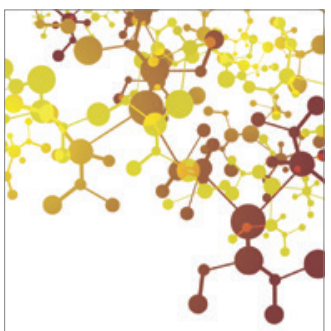

Journal of

Applied Chemistry

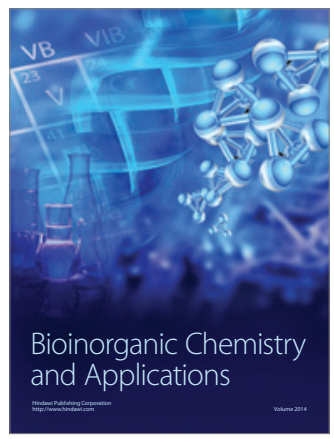

Inorganic Chemistry
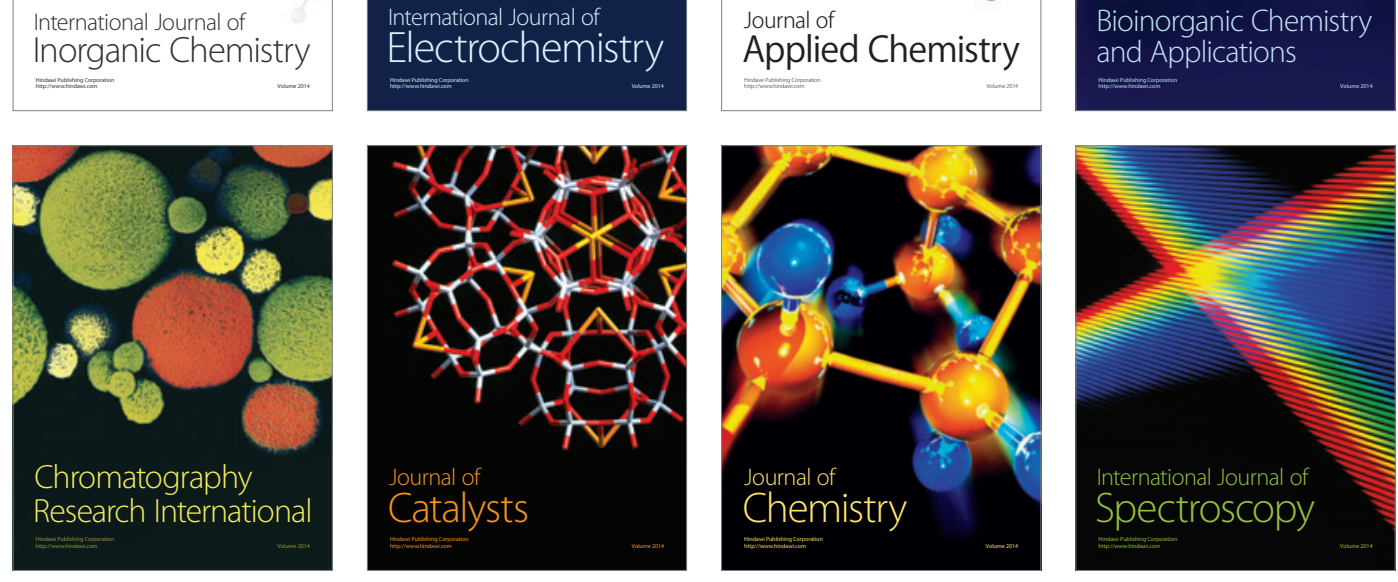